Diel R, Rutz S, Castell S, et al. Tuberculosis: cost of illness in Germany. Eur Respir J 2012; 40: $143-151$.

Sotgiu G, Centis R, D’Ambrosio L, et al. Efficacy, safety and tolerability of linezolid containing regimens in treating MDR-TB and XDR-TB: systematic review and meta-analysis. Eur Respir J 2012; 40: 1430-1442.

Migliori GB, Sotgiu G. Treatment of tuberculosis: have we turned the corner? Lancet 2012; 380: 955-957.

De Lorenzo S, Alffenaar JW, Sotgiu G, et al. Efficacy and safety of meropenem/clavunate added to linezolid containing regimens in the treatment of M/XDR-TB. Eur Respir J 2013; 41: 1386-1392.

8 Skripconoka V, Danilovits M, Pehme L, et al. Delamanid improves outcomes and reduces mortality in multidrugresistant tuberculosis. Eur Respir J 2013; 41: 1393-1400.

9 World Health Organization. The use of bedaquiline in the treatment of multidrug-resistant tuberculosis: interim policy guidance. Document WHO/HTM/TB/2013.6. Geneva, World Health Organization, 2013.

10 Horsburgh CR Jr, Haxaire-Theeuwes M, Lienhardt C, et al. Compassionate use of and expanded access to new drugs for drug-resistant tuberculosis. Int J Tuberc Lung Dis 2013; 17: 146-152.

\title{
Extensively drug-resistant tuberculosis: early access to bedaquiline for a UK patient
}

\author{
To the Editor:
}

The prevalence of drug resistance among tuberculosis (TB) cases is increasing in the UK [1] and worldwide [2], with increasing proportions of cases with multidrug- and extensively drug-resistant TB (MDR-/XDRTB) being reported $[1,2]$. Toxicity and poor efficacy of treatment are major problems and long durations with culture-positive disease increase the risk of transmission. This report describes difficulties in managing XDR-TB and the pre-license use of bedaquiline outside of clinical trials.

Bedaquiline is an anti-tuberculous drug of the diarylquinoline class, which inhibits bacterial adenosine triphosphate synthase, and represents the first new class of anti-tuberculous agents for at least four decades. Its unique mechanism means that there is no cross-resistance with other drugs in current use. In phase II clinical trials, in combination with background MDR-TB regimens, bedaquiline resulted in reduced time to sputum culture conversion, reduced emergence of resistance to companion drugs and little additional toxicity compared with background regimens alone [3]. At present it is not yet licensed in Europe and the World Health Organization (WHO) has recently issued interim policy guidance for its use as part of combination therapy for adults with MDR-TB under specific conditions, based on currently available evidence [3].

A 28-year-old, HIV-negative Indian female with no prior history of TB treatment or contact was diagnosed with TB through screening on arrival in the UK. A pre-departure chest radiograph 3 months before assessment in the UK was reported to be normal. A bacille Calmette-Guérin scar was seen on examination. A tuberculin skin test and interferon- $\gamma$ release assay were positive and she was asymptomatic. Chest radiograph revealed mediastinal lymphadenopathy and 1 month later she developed cough and fever. Sputum specimens were sent for microscopy and culture, and further imaging was requested.

Computed tomography (CT) imaging confirmed thoracic lymphadenopathy, and showed small pericardial and pleural effusions and a small pulmonary nodule. Mycobacterium tuberculosis was isolated on culture from smear-negative sputum specimens. The identification of the organisms was confirmed by GenoType DNA strip (Hain Lifescience, Nehren, Germany), but no molecular tests for resistance were requested by the referring centre. Standard quadruple therapy (rifampicin, isoniazid, pyrazinamide and ethambutol) was commenced in May 2011 (week 1) (fig. 1), while drug susceptibility testing (DST) was in progress and the appropriate contact tracing procedure was initiated.

After 3 weeks of quadruple therapy, first-line DST showed resistance to rifampicin, isoniazid and ethambutol. The patient was transferred to the regional infectious diseases unit (Dept of Infectious Diseases and Tropical Medicine (Monsall Unit), North Manchester General Hospital, Manchester, UK) and hospitalised to commence treatment for MDR-TB with pyrazinamide, moxifloxacin, amikacin, prothionamide, cycloserine and azithromycin (fig. 1). Pericardial involvement was confirmed by 


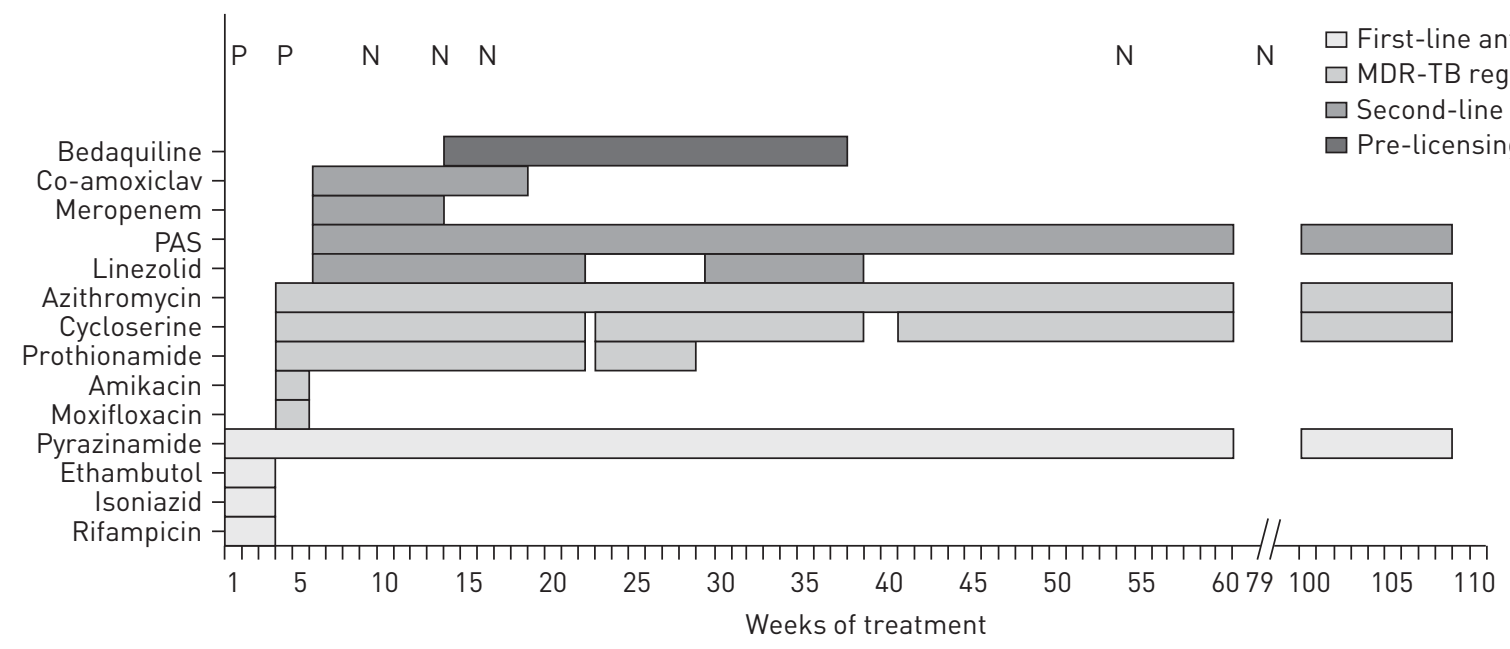

FIGURE 1 Schematic diagram showing the treatment course in this case of extensively drug-resistant tuberculosis. MDR-TB: multidrug-resistant tuberculosis; WHO: World Health Organization; PAS: para-amino salicylic acid; P: positive sputum culture; N: negative sputum culture.

echocardiogram and review of CT images. Corticosteroids were therefore added according to national guidelines [4] and reduced over a period of 8 weeks.

At week 5, DST showed resistance to fluoroquinolones and the injectable agents streptomycin, amikacin, capreomycin and kanamycin, confirming XDR-TB [2], plus additional resistance to all injectables and ethambutol, which is associated with poorer outcomes $[5,6]$. The isolate was susceptible to pyrazinamide, prothionamide, linezolid and the macrolides azithromycin and clarithromycin. Therefore, moxifloxacin and amikacin were discontinued, and linezolid [7], para-amino salicylic acid (PAS), meropenem [8] and coamoxiclav (for clavulanic acid) were added at week 5. Treatment with pyrazinamide, prothionamide, cycloserine and azithromycin was continued. At week 10, the isolate was reported to be susceptible to PAS. Cycloserine susceptibility testing is not available in the UK.

With full DST results and limited therapeutic options, an application was made for access to bedaquiline through the manufacturer's compassionate use programme. Access was granted for this patient who had primarily pulmonary disease. Bedaquiline was commenced in August 2011 at week 14 (fig. 1), using recommended doses of $400 \mathrm{mg}$ daily for 2 weeks, then $200 \mathrm{mg}$ three times a week for 22 weeks, as this is the maximum duration permitted based on current evidence [3].

Regular sputum sampling was undertaken and mycobacterial cultures were negative from week 9 (5 weeks before starting bedaquiline), throughout treatment.

As standard practice, and as part of the requirement for bedaquiline, toxicity monitoring was undertaken. ECGs were initially performed daily, in view of the combination of bedaquiline with azithromycin, and then less frequently as no QT prolongation was detected. Transaminase levels, amylase and renal function were monitored, along with frequent symptomatic enquiry. Toxicity problems were experienced during therapy, particularly nausea and a painful, progressive peripheral neuropathy, which led to stopping linezolid at week 22, interruption of cycloserine, prothionamide and azithromycin for 3 days at week 23, later interruption of cycloserine for 3 weeks (fig. 1), and subsequent withdrawal of prothionamide due to intolerable peripheral neuropathy, which worsened on re-challenge with prothionamide, despite high-dose pyridoxine throughout. Hypothyroidism secondary to PAS was treated with thyroxine from week 9 (week 4 of PAS). She complained of vertigo and tinnitus from week 70. Transaminase levels were normal throughout. No specific adverse events were attributed to bedaquiline and no interruption of bedaquiline was required. The patient was discharged from hospital after 5 weeks of bedaquiline (week 19), with normal ECGs and negative sputum cultures. Outpatient therapy was not directly observed after careful consideration of risk for non-adherence over many weeks. Treatment was discussed with a member of the British Thoracic Society MDR-TB Clinical Advice Service.

Follow-up CT scans showed improvement of mediastinal lymphadenopathy and resolution of effusions at weeks 15 and 33, and normalisation of mediastinal appearance with complete resolution of the pulmonary nodule at week 63. The patient remains culture negative, having culture converted within 6 weeks of 
commencing treatment for MDR-TB (week 9 of treatment) (fig. 1), and has completed her planned 2-year treatment regimen. Follow-up is ongoing.

This case of XDR-TB in an individual with no previous history of TB treatment demonstrates the need for vigilance and adequate sampling for mycobacterial culture before initiating anti-tuberculous treatment. For this individual, born in a high TB-incidence country, the diagnosis and effective treatment of XDR-TB were delayed by the omission to request molecular tests for drug resistance on the first positive sputum specimen. Although the isolate was susceptible to prothionamide, its continued use was ultimately precluded by a progressive, painful neuropathy. Treatment of drug-resistant TB carries a risk of treatment failure and toxicity, as the drugs used are often less effective than first-line treatment and the long duration required means that patients are exposed to a high risk of toxicity. Following this case we have streamlined procedures for requests to reference laboratories so that all DST is requested simultaneously for MDR-TB.

Bedaquiline, added to background regimens for drug-resistant TB, increased treatment efficacy in phase II trials and results of phase III trials are awaited to confirm safety and efficacy. The most commonly reported side-effect in trials was nausea and some prolongation of the QT interval has been reported [3]. Its lack of toxicity in this case meant that no treatment interruption was required, in contrast with other more toxic drugs in this regimen, and it is likely to have contributed to a successful outcome.

European licensing of bedaquiline is much anticipated for those treating, and being treated for, drug-resistant TB. New drugs may contribute to the development of shorter, less toxic treatment regimens. Further toxicity and efficacy data for bedaquiline are awaited from larger trials and post-marketing surveillance. Since 2012, clinicians may benefit from the European Standards for Tuberculosis Care [9] and the European Respiratory Society/WHO Consilium [10], which offer guidance on managing this challenging infection.

0 @ERSpublications

XDR-TB: pre-licensing experience with bedaquiline contributed to a successful outcome without additional toxicity http://ow.ly/pMX6y

Clare van Halsema ${ }^{1}$, Susan Humphreys ${ }^{1}$ and Alec Bonington ${ }^{1,2}$

${ }^{1}$ Dept of Infectious Diseases and Tropical Medicine (Monsall Unit), North Manchester General Hospital, Manchester, and ${ }^{2}$ Manchester Academic Health Science Centre, The University of Manchester, North Manchester General Hospital, Manchester, UK

Correspondence: C. van Halsema, Dept of Infectious Diseases and Tropical Medicine (Monsall Unit), North Manchester General Hospital, Delaunays Road, Manchester, M8 5RB, UK. E-mail: claretaylor@doctors.org.uk

Received: July 262013 | Accepted: Aug 082013 | First published online: Aug 292013

Conflict of interest: None declared.

\section{References}

1 Health Protection Agency. Tuberculosis in the UK: annual report on tuberculosis surveillance in the UK, 2012. London, Health Protection Agency, 2012.

World Health Organization. Anti-tuberculosis drug resistance in the world. Report No. 4. Geneva, WHO, 2008. World Health Organization. The use of bedaquiline in the treatment of multidrug-resistant tuberculosis. Interim policy guidance. Geneva, WHO, 2013.

4 National Institute for Health and Clinical Excellence. Clinical diagnosis and management of tuberculosis and measures for its prevention and control. NICE clinical guideline 117. London, NICE, 2011.

5 Falzon D, Gandhi N, Migliori GB, et al. Resistance to fluoroquinolones and second-line injectable drugs: impact on multidrug-resistant TB outcomes. Eur Respir J 2013; 42: 156-168.

6 Migliori GB, Sotgiu G, Gandhi NR, et al. Drug resistance beyond extensively drug-resistant tuberculosis: individual patient data meta-analysis. Eur Respir J 2013; 42: 169-179.

7 Sotgiu G, Centis R, D’Ambrosio L, et al. Efficacy, safety and tolierability of linezolid containing regimens in treating MDR-TB and XDR-TB: systematic review and meta-analysis. Eur Respir J 2012; 40: 1430-1442.

8 De Lorenzo S, Alffenaar JW, Sotgiu G, et al. Efficacy and safety of meropenem-clavulanate added to linezolidcontaining regimens in the treatment of MDR-/XDR-TB. Eur Respir J 2013; 41: 1386-1392.

9 Migliori GB, Zellweger I, Abubakar E, et al. European union standards for tuberculosis care. Eur Respir J 2012; 39: 807-819.

10 Blasi F, Dara M, van der Werf MJ, et al. Supporting TB clinicians managing difficult cases: the ERS/WHO Consilium. Eur Respir J 2013; 41: 491-494. 